\title{
Editorial: Advances on Bio-inspired Information, Communication and Computational Systems
}

\author{
Junichi Suzuki $^{1} \cdot$ Parisa Memarmoshrefi $^{2} \cdot$ Tadashi Nakano $^{3}$
}

Published online: 16 March 2016

(C) Springer Science+Business Media New York 2016

\section{Editorial:}

This special issue features seven selected papers from the 8th International Conference Bio-inspired Information and Communications Technologies (BICT), which took place in Boston, MA, USA, from December 1 to 3, 2014. These papers report the recent advances in bio-inspired information, communication and computational systems, addressing important and emerging research issues and covering novel solutions in analysis/design methodologies, algorithms and applications.

The first paper entitled "Epistatic Signaling and Minority Games, the Adversarial Dynamics in Social Technological Systems" shows a natural role for signaling games in modeling various strategic interactions among agents in a socialtechnological network such as an internet of humans and things (IOHT); with the aim of study of the behavior of the entity in the network such as honest and deceptive behavior. In particular, the effect of augmenting the two-player sender-receiver games with a recommendation-verification system and a newly devised crypto-coin (e.g., M-Coin) is investigated. The paper design a signaling game approach called epistatic signaling and explored the role of minority games in this context. The simulations results have identified some behaviors such as the behavior of the attackers in using the signal complexity as the dimension of the attack.

Junichi Suzuki

jxs@cs.umb.edu

1 University of Massachusetts, Boston, Boston, MA, USA

2 Institute of Computer Science, University of Göttingen, Göttingen, Germany

3 Graduate School of Frontier Biosciences, Osaka University, Osaka, Japan
The second paper, "Bioinspired Security Analysis of Wireless Protocols," investigates feasibility of adopting fraglets as model for specifying security protocols and analysing their properties. Fraglets represent an execution model for communication protocols that resembles the chemical reactions in living organisms. They are computation fragments flowing through a computer network. The strong connection between their way of transforming and reacting and formal rewriting systems makes a fraglet program amenable to automatic verification. The paper shows a concrete sample analyses over a secure RFID protocol. The results of analysis confirm the effectiveness of the cryptofraglets framework for the model and analysis of security properties.

The next paper, "Biometric Inspired Mobile Network Authentication and Protocol Validation," improves the existing biometric authentication protocol by validation of these protocol and assessing their security level. As one of the technological application of biometric authentication, authentication in Wireless Body Area Networks is focused in this work; where tiny sensors on body need new security mechanisms rather than traditional cryptographically-based approaches. In the work two-tier authentication is proposed. Security in the first tier, on-body nodes physiological authentication, is based on agreeing secrete key (U-key) based on the unique physiological features. The second tire is to securing data communication between on-body sensors and the server based on public key cryptography. However, by validating the model, authors suggest the use of asymmetric encryption, like AES, to encrypt the data with a random key and the use of asymmetric encryption, like RSA, to encrypt that random key for encrypting data between on body network and the server. This will benefit the protocol by giving it the ability to send a bigger amount of data if needed, especially the public key of the client. Complexity approximation is presented in the work as well. 
The article entitled "Autonomous Ant-based Public Key Authentication Mechanism for Mobile Ad-hoc Networks" proposed a self-organized and localized public key authentication mechanism borrowed from nature and it is based on ant colony systems. Every node generates its own public-private key pair, issues certificates to neighboring nodes and provides on-demand authentication services by means of gathering certificate chains towards a target node. Pheromone concentration left by ants along the path of the certificate chains represents the trust level of a node towards other nodes. This model is able to authenticate public keys by selecting the most trustworthy certificate chains gathered by ants and can identify and exclude certificate chains with malicious nodes. Simulation results demonstrate that, despite of having malicious intermediate nodes in the network who relay the false certificate, the model is suitable for static networks and networks with low to high mobility.

The next paper, "The structural role of feed-forward loop motif in gene regulatory networks," leverages machine learning methods to understand the contributing factors to the robustness of biological networks, particularly gene regulatory networks. It examines various features based on structural/ topological significance, such as transcriptional motifs, and other traditional metrics, such as network density and average shortest path, and identifies feed-forward loop based features as important contributors to the robustness of transcriptional networks extracted from the bacterium Escherichia coli and the baker's yeast Saccharomyces cerevisiae, at different perturbation levels. The proposed machine learning methods also reveal the role of feed forward loops in signal transduction in the Escherichia coli regulatory networks. This finding has substantial impacts on designing engineered systems (e.g., wireless sensor networks) that exploit the topological properties of biological networks to maximize efficiency and robustness.

The article entitled "Contagion of Evacuation Decision Making on Real Map" studies multi-agent modeling of individual residents affected by natural disasters such as an earthquake and a subsequent tsunami. It assumes that agents possess psychological conditions, make evacuation decisions through interacting nearby agents and move toward shelters. Simulation results reveal how the structure of social networks and the contagion of evacuation decision making impact collective behaviors of agents with real geographical data.

The last paper, "R2 Indicator based Evolutionary Multiobjective Optimization for the Pickup and Delivery Problem with Time Windows and Demands," formulates a multiobjective variant of the Pickup and Delivery Problem (PDP), called PDP with Time Windows and Demands (PDP-TW-D), and attempts to solve the problem with an evolutionary optimization algorithm. With respect to conflicting five optimization objectives including the number of vehicles and the total travel distance, the goal of PDP-TW-D is to find a set of Pareto-optimal routes for a fleet of vehicles in order to serve given transportation requests. This problem is well applicable to operational optimization requirements in various real-world mobile network systems (e.g., fleet operation of wirelessly-connected mobile robots, drones and autonomous vehicles). The proposed algorithm integrates global and local search processes to improve convergence speed and effectively obtains quality trade-off solutions with respect to conflicting objectives.

The guest editors appreciate reviewers for their efforts in reviewing manuscripts. We are also grateful to Drs. Imrich Chlamtac and Sara Fruner for their significant guidance to edit this special issue.

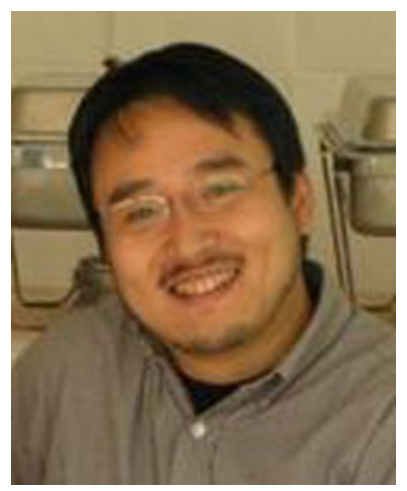

Junichi Suzuki is an Associate Professor of Computer Science at the University of Massachusetts, Boston (UMass Boston). He is the director of UMass Boston's Distributed Software Systems Group (2004 ) and a co-director of UMass Boston's Center for Coastal Environmental Sensing Networks (2007 ). He is a recipient of the Dean's Outstanding Research Achievement Award in UMass Boston's College of Science and Mathematics (2008).

He received a Ph.D. in Computer Science from Keio University, Japan, in 2001. He was a postdoctoral research fellow at the University of California, Irvine (UCI) from 2001 to 2004. Before joining UCI, he was with Object Management Group Japan, Inc., as Technical Director.

His research interests include biologically-inspired computing/networking, autonomous and adaptive distributed systems, body area networks, molecular communication, sustainable networking and multiobjective optimization. In these areas, he has authored three books, edited three books, edited 15 journal special issues, and published one industrial standard specification and $140+$ papers in international journals and conferences. He is a recipient of eight best paper awards and two best poster awards at major conferences. He has delivered keynote speeches at six conferences such as IEEE SSCI 2013 and IES 2011. He serves on the editorial boards for eight international journals including Springer Journal on Complex Adaptive Systems Modeling and Elsevier Nano Communication Networks Journal. He has chaired or co-chaired 25 international conferences such as BICT 2015-2014, IEEE HealthCom 2015, IEEE PIMRC 2014, BodyNets 2014-2012, BIONETICS 2010 and ICSOC 2009. He has served on the steering committee of 10 conferences as well as the program committee of $150+$ conferences such as IEEE GLOBECOM, IEEE SECON, IEEE SASO, IEEE CEC, ACM GECCO, IEEE ICTAI and ACM/IEEE BIOSIGNALS. He has served as a panelist for the U.S. National Science Foundation, the U.S. Army Corps of Engineers Engineer Research and Development Center, the European Commission and the Israel Science Foundation to review grant proposals. He is a member of ISO/IEC JTC1-SC7 (Software and System Engineering) and Object Management Group's Super Distributed Objects SIG. 


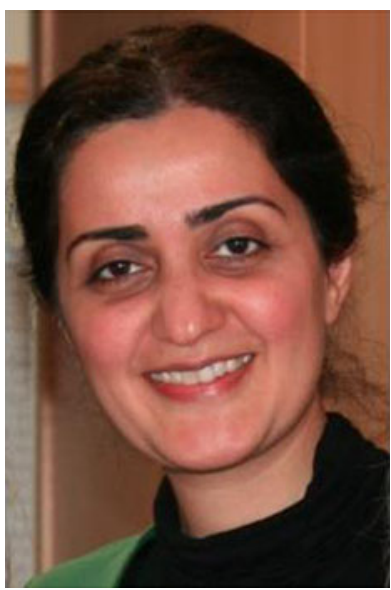

Parisa Memarmoshrefi is a Postdoctoral research fellow in computer science at University of Göttingen, Germany since Jun 2012. She received a $\mathrm{PhD}$ in Computer Science, Telematics group, at University of Göttingen in 2012. Her research interests include security in wireless network such as ad-hoc and sensor networks, self-organized and distributed authentication mechanisms, biologically-inspired computing such as swarm intelligent, and soft security mechanism such as trustbased systems. She has published several papers in these areas and among them she received best paper awards at conferences: BICT (Bionetics) and WiMob.

She has served as the reviewer of several conference papers such as in BodyNet, Embedded and Ubiquitous Computing (EUC) and Journal papers such as in International Journal of Ad Hoc and Ubiquitous Computing (IJAHUC) and the Computer Journal. She has chaired special track workshop and served as the TPC in conferences: BICT and MOWNET.

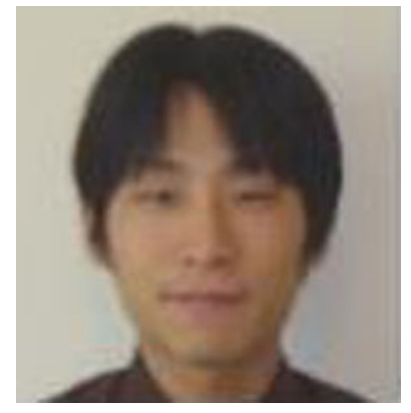

Tadashi Nakano is an Associate Adjunct Professor of the Institute for Academic Initiatives, Osaka University, and a Visiting Associate Professor of the Graduate School of Biological Sciences, Osaka University. Dr. Nakano has been engaged in research at the intersection of computer science and biology, including design, implementation, and evaluation of molecular communication systems, synthetic biological systems, and biologically inspired systems. His research interests also include network applications and distributed computing systems with strong emphasis on interdisciplinary approaches. 